\title{
The population impact of rheumatic and musculoskeletal diseases in relation to other non-communicable disorders: comparing two estimation approaches
}

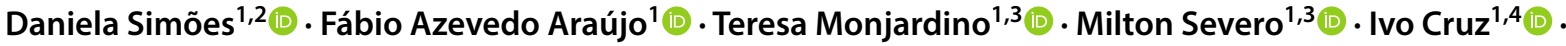 \\ Loreto Carmona ${ }^{5}$ (i) $\cdot$ Raquel Lucas $^{1,3}$ (i)
}

Received: 18 December 2017 / Accepted: 29 January 2018 / Published online: 8 February 2018

(c) Springer-Verlag GmbH Germany, part of Springer Nature 2018

\begin{abstract}
The aim of this study was to quantify the population impact of rheumatic and musculoskeletal diseases (RMDs) with other non-communicable diseases (NCDs), using two complementary strategies: standard multivariate models based on global burden of disease (GBD)-defined groups vs. empirical mutually exclusive patterns of NCDs. We used cross-sectional data from the Portuguese Fourth National Health Survey $(n=23,752)$. Six GBD-defined groups were included: RMDs, chronic obstructive pulmonary disease or asthma, cancer, depression, diabetes or renal failure, and stroke or myocardial infarction. The empirical approach comprised the patterns "low disease probability", "cardiometabolic conditions", "respiratory conditions" and "RMDs and depression". As recommended by the outcome measures in rheumatology (OMERACT) initiative, health outcomes included life impact, pathophysiological manifestations, and resource use indicators. Population attributable fractions (PAF) were computed for each outcome and bootstrap confidence intervals (95\% CI) were estimated. Among GBDdefined groups, RMDs had the highest impact across all the adverse health outcomes, from frequent healthcare utilization (PAF 7.8\%, 95\% CI 6.2-9.3) to negative self-rated health (PAF 18.1\%, 95\% CI 15.4-20.6). In the empirical approach, patterns "cardiometabolic conditions" and "RMDs and depression" had similar PAF estimates across all adverse health outcomes, but "RMDs and depression" showed significantly higher impact on chronic pain (PAF 8.9\%, 95\% CI 7.6-10.3) than the remaining multimorbidity patterns. RMDs revealed the greatest population impact across all adverse health outcomes tested, using both approaches. Empirical patterns are particularly interesting to evaluate the impact of RMDs in the context of their co-occurrence with other NCDs.
\end{abstract}

Keywords Rheumatic diseases · Musculoskeletal diseases · Non-communicable diseases $\cdot$ Multimorbidity $\cdot$ Public health · Health impact assessment

Raquel Lucas

rlucas@med.up.pt

Daniela Simões

daniela.simoes@ispup.up.pt

Fábio Azevedo Araújo

fabio.araujo@ispup.up.pt

Teresa Monjardino

teresam@med.up.pt

Milton Severo

milton@med.up.pt

Ivo Cruz

icruz@arsnorte.min-saude.pt
Loreto Carmona

loreto.carmona@inmusc.eu

1 EPIUnit-Instituto de Saúde Pública, Universidade do Porto, Rua das Taipas, 135-139, 4050-600 Porto, Portugal

2 Escola Superior de Saúde de Santa Maria, Porto, Portugal

3 Departamento de Ciências da Saúde Pública e Forenses e Educação Médica, Faculdade de Medicina, Universidade do Porto, Porto, Portugal

4 Unidade de Saúde Pública do ACeS Grande Porto V-Porto Ocidental, Porto, Portugal

5 Instituto de Salud Musculoesqueletica, Madrid, Spain 


\section{Introduction}

Rheumatic and musculoskeletal diseases (RMDs) are a highly prevalent and heterogeneous group of non-communicable diseases (NCDs) [1]. Despite their well-known association with a wide scope of adverse health outcomes [2-5], the comparative burden of RMDs at the populationlevel in relation to other NCDs is insufficiently explored, probably because they have a low case-fatality.

Population attributable fractions (PAF) are an important epidemiological measure to estimate impact because they combine both the prevalence of disease in a target population and the strength of the associations between that condition and each of its outcomes. Under certain assumptions, they provide an estimate of the proportion of the burden of each outcome that could be prevented if the corresponding disease was eliminated from the population [6]. The confrontation between individual consequences (i.e., strength of associations) and population-level impact (i.e., PAF) demonstrate how the determinants of clinical priorities may, or may not, conflict with those of population-based priorities in public health policy.

To ensure feasibility at the global scale, the global burden of disease (GBD) study focuses on functional domains of health, such as body functions, structures, and complex human operations, to the detriment of broader aspects of human health such as well-being and economic impact, which require more specific investigations [7]. We believe that the comparative impact of RMDs in relation to other NCDs with regard to a wide variety of adverse outcomes deserves to be specifically examined. The outcome measures in rheumatology (OMERACT) initiative has proposed a core set of measures to provide consistent and comparable estimates of disease burden and fully address the impact of RMDs [8, 9]. PAF estimates computed for the core set of outcome measures can be immediately useful for enriching the public health policy debate and can help as a comprehensive starting point to provide in-depth population awareness about the importance of RMDs prevention and management.

Due to their high incidence and low case-fatality, RMDs frequently co-exist with other conditions, a phenomenon known as multimorbidity [10]. Multimorbidity poses a technical challenge when the aim is to disentangle and estimate the comparative burden of RMDs in the context of all NCDs in the population. Additionally, multimorbidity might lead to the overestimation of the burden of each individual NCD [11]. Despite this well-known problem, several studies attempt to isolate independent effects of each disease through standard multivariate adjustment, which may not provide a realistic depiction of the multimorbidity burden $[2,3]$.
To address that, in the present paper, we use two complementary strategies to quantify and compare the population impact of NCDs with a focus on the contribution of RMDs: standard multivariate models based on GBD-defined groups of diseases vs. empirical mutually exclusive patterns of NCDs. We estimate impact across several adverse health outcomes that fulfill the OMERACT criteria.

\section{Methods}

\section{Fourth Portuguese national health survey}

The Portuguese national health survey (INS) is a government-sponsored periodic nationwide health survey conducted by statistics Portugal (INE, IP) and the National Institute of Health Doutor Ricardo Jorge [12]. In the 2005/6 survey (IV-INS), a representative sample of the Portuguese population was obtained through complex stratified and cluster sampling [12]. Initially, a sample of households was defined, using the 2001 Population and Housing Census as the sampling frame. Within each main geographical region, two strata were defined: the parishes and, within each parish, geographically defined units of approximately 240 households. A random sample of households was selected within each geographically defined unit and all persons living in those households were surveyed. People living in collective residential institutions at the time of recruitment were not eligible. The survey was carried out in compliance with the Helsinki Declaration. The sample size was defined to ensure homogeneous distribution of the participants by the seven level II nomenclature of territorial units for statistics (NUTS II) regions.

Between February 2005 and January 2006, trained interviewers evaluated, through computer-assisted interviews, 41,193 subjects from 15,239 different households (76\% participation at household level). Data analysis was restricted to participants aged $\geq 15$ years, corresponding to a sample of 35,229 respondents. Information was collected directly from selected individuals or from a proxy respondent, i.e., another adult member of the household. Due to limitations in the validity of information on health conditions and outcomes, all individuals whose answers were obtained from proxy respondents (11,388 interviews; $32.3 \%)$ were excluded from the present analysis, yielding a sample of 23,841 respondents. Additionally, 89 subjects were excluded from the analysis due to missing information in at least one of the NCDs. The final sample comprised 23,752 participants.

For the present analysis, we selected the following background variables: sex, age and education in years of completed schooling. Participants were grouped into five age categories: $18-34,35-54,55-64,65-79$ or $\geq 80$ years; and 
five education categories: no formal education, 1-4, 5-9, $10-12$ or $\geq 13$ years of schooling.

\section{Non-communicable diseases}

Among the diseases inquired in the IV-INS survey, we selected those that were common to the United States Department of Health and Human Services-Office of the Assistant Secretary of Health (OASH) list of conditions that meet the definition of chronicity, are highly prevalent, and/or are amenable to public health or clinical interventions [13]: RMDs, diabetes, hypertension, chronic obstructive pulmonary disease (COPD), stroke, depression, myocardial infarction, cancer, osteoporosis, asthma, and renal failure. Each was considered present if participants reported a previous diagnosis by a physician or nurse.

Two approaches for the aggregation of diseases were used: (a) GBD-defined groups of diseases adjusted for the remaining diseases, and (b) empirical mutually exclusive patterns of multimorbidity from NCDs. In the first approach, conditions were aggregated into one of six groups according to GBD grouping: (1) RMDs, (2) COPD or asthma, (3) cancer, (4) depression, (5) diabetes or renal failure, and (6) stroke or myocardial infarction [14]. In the second approach, we applied our previously defined model-based patterns of NCD co-occurrence to define exposures [15]. In that work, we used latent class analysis to identify patterns of coexistence of 11 chronic non-communicable diseases (RMDs, diabetes, hypertension, chronic obstructive pulmonary disease, stroke, depression, myocardial infarction, cancer, osteoporosis, asthma, and renal failure). After selecting the number of latent classes (also referred to as patterns) among those with the lowest Bayesian Information Criterion (BIC), we found that multimorbidity in the Portuguese population could be summarized into four patterns of chronic non-communicable diseases co-occurrence, that were labeled according to disease frequency as "low disease probability" (reference pattern), "cardiometabolic conditions", "respiratory conditions" and "RMDs and depression". We also found that RMDs were highly prevalent across all multimorbidity patterns: $38.6 \%$ in "cardiometabolic conditions", $53.5 \%$ in "respiratory conditions" and $66.7 \%$ in "RMDs and depression", while only $7.8 \%$ in "low disease probability" pattern [15].

\section{Adverse health outcomes}

According to the OMERACT core areas of outcomes for rheumatologic conditions [8,9], we analyzed a large set of outcomes, including life impact (self-rated health, and shortand long-term disability), pathophysiological manifestations (chronic pain) and resource use indicators (healthcare utilization and out-of-pocket healthcare expenses), all available in the IV-INS survey. All measures of health-related dimensions were dichotomized to define the presence or absence of an adverse health outcome, as previously described in detail [15].

\section{Statistical analysis}

To obtain representative estimates for the Portuguese population, sampling weights were applied to all statistical analyses. Weights were provided by Statistics Portugal along with the IV-INS database, and were computed based on the inverse of the probability of selection of each sampling unit and further corrected for non-response and effective number of subjects evaluated, regarding age and sex [12].

Sample characteristics are presented as counts and proportions (unweighted and weighted). The associations between GBD-defined groups or empirical model-based patterns of NCDs and the presence of adverse health outcomes were estimated through adjusted prevalence ratios (adjPR) with 95\% confidence intervals (95\% CI), computed using multivariate log-Poisson regression analyses. In the first approach, the associations of each one of the six groups of diseases with the relevant outcomes were estimated after adjustment for sex, age and education, and also for the remaining five groups of diseases to account for multimorbidity, as previously performed [2]. In the second approach, since patterns themselves represent multimorbidity, estimates were adjusted only for sex, age and education.

PAF were computed through the formula: $p_{(\text {o) }} \times$ [(adjPR -1$) / \operatorname{adjPR}]$, where $p_{(\mathrm{o})}$ is the proportion of individuals with the adverse health outcome that reported having each NCD or were assigned to each pattern. For the GBDdefined groups approach, each resulting PAF can be defined as the proportion of cases reporting the adverse health outcome that would be prevented following elimination of that chronic condition or group of conditions, assuming that the distributions of the other diseases remained unchanged. When addressing empirical patterns, PAF indicates the proportion of cases that would be prevented by changing class membership into the "low disease probability" pattern. We provide bootstrap-confidence intervals $(95 \% \mathrm{CI})$, for each PAF estimate, calculated using a set of 1000 replications [16].

Statistical analyses were performed in R language and software environment for statistical computation version 3.1.3 (R Foundation for Statistic Computing, Austria).

\section{Results}

Table 1 summarizes the characteristics of participants. Weighted distribution provided a sample where most subjects were female $(52.1 \%)$, and over two-thirds were between 18 and 54 years of age and had only nine or less 
Table 1 Sociodemographic characteristics, non-communicable diseases, patterns of non-communicable diseases and adverse health outcomes in the general population

\begin{tabular}{|c|c|c|c|}
\hline & $n$ & Unweighted \% & Weighted \% \\
\hline \multicolumn{4}{|l|}{ Sex } \\
\hline Female & 14,069 & 59.2 & 52.1 \\
\hline Male & 9683 & 40.8 & 47.9 \\
\hline \multicolumn{4}{|l|}{ Age, years } \\
\hline $18-34$ & 4775 & 20.1 & 33.3 \\
\hline $35-54$ & 8124 & 34.2 & 33.4 \\
\hline $55-64$ & 3971 & 16.7 & 13.2 \\
\hline $65-79$ & 5641 & 23.8 & 15.6 \\
\hline$\geq 80$ & 1241 & 5.2 & 4.6 \\
\hline \multicolumn{4}{|l|}{ Education, years } \\
\hline No formal education & 4026 & 17.0 & 11.4 \\
\hline $1-4$ & 8815 & 37.1 & 30.2 \\
\hline $5-9$ & 5766 & 24.3 & 29.3 \\
\hline $10-12$ & 2934 & 12.4 & 16.4 \\
\hline$\geq 13$ & 2211 & 9.3 & 12.8 \\
\hline \multicolumn{4}{|c|}{ Non-communicable diseases (presence) } \\
\hline RMDs & 5075 & 21.4 & 18.1 \\
\hline Diabetes & 2323 & 9.8 & 7.8 \\
\hline Hypertension & 7061 & 29.7 & 24.2 \\
\hline COPD & 907 & 3.8 & 4.3 \\
\hline Stroke & 531 & 2.2 & 1.8 \\
\hline Depression & 2338 & 9.8 & 9.8 \\
\hline Myocardial infarction & 453 & 1.9 & 1.5 \\
\hline Cancer & 625 & 2.6 & 2.3 \\
\hline Asthma & 1316 & 5.5 & 5.5 \\
\hline Renal failure & 405 & 1.7 & 1.6 \\
\hline Osteoporosis & 2289 & 9.6 & 7.3 \\
\hline \multicolumn{4}{|c|}{ Patterns of non-communicable diseases } \\
\hline Low disease probability & 17,936 & 75.5 & 80.0 \\
\hline Cardiometabolic conditions & 3086 & 13.0 & 10.3 \\
\hline Respiratory conditions & 476 & 2.0 & 2.1 \\
\hline RMDs and depression & 2254 & 9.5 & 7.6 \\
\hline \multicolumn{4}{|c|}{ Adverse health outcomes (presence) } \\
\hline Negative self-rated health & 4644 & 19.6 & 16.0 \\
\hline Short-term disability & 2562 & 10.8 & 11.6 \\
\hline Long-term disability $^{\mathrm{a}}$ & 1440 & 22.8 & 21.6 \\
\hline Chronic pain & 4686 & 19.7 & 19.9 \\
\hline $\begin{array}{l}\text { Frequent healthcare utiliza- } \\
\text { tion }\end{array}$ & 6795 & 28.6 & 29.0 \\
\hline $\begin{array}{l}\text { Out-of-pocket healthcare } \\
\text { expenses }\end{array}$ & 6099 & 25.7 & 22.8 \\
\hline
\end{tabular}

Fourth national health survey (IV-INS), Portugal, 2005-2006

$R M D s$ rheumatic and musculoskeletal diseases, COPD chronic obstructive pulmonary disease

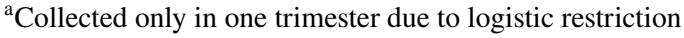

years of education. Hypertension was the most frequently reported condition (24.2\%), followed by RMDs. Frequent healthcare utilization was the most frequently reported adverse health outcome $(29.0 \%)$, followed by out-ofpocket healthcare expenses (22.8\%), long-term disability (21.6\%), and chronic pain (19.9\%).

As presented in Fig. 1, RMDs had the highest prevalence of all NCDs across all the adverse health outcomes (from $29.5 \%$ in people with frequent healthcare utilization to $47.3 \%$ in people with negative self-rated health). RMDs had the strongest association with chronic pain (adjPR 1.69, 95\% CI 1.57-1.82), while depression had the strongest association with all the remaining adverse health outcomes. At the population-level and after adjustment for the remaining conditions, RMDs had the highest population impact across all the adverse health outcomes, with PAF varying from 7.8\% (95\% CI 6.2-9.3) in frequent healthcare utilization to $18.1 \%$ (95\% CI 15.4-20.6) in negative self-rated health (Fig. 2). The impact of RMDs on negative self-rated health, long-term disability and chronic pain was significantly higher than the one associated with other conditions. RMDs were followed by depression (PAF ranging from $5.7 \%, 95 \%$ CI 3.6-8.0 in long-term disability to $11.0 \%, 95 \%$ CI 9.3-12.6 in negative self-rated health), and then by diabetes and renal failure. Indeed, the impact of the RMDs on short-term disability, frequent healthcare utilization and healthcare utilization was similar to the impact of depression.

When considering empirical model-based patterns of NCDs (Fig. 3), the "cardiometabolic conditions" pattern was the most prevalent across all adverse health outcomes, closely followed by "RMDs and depression" pattern (ranging from $14.7 \%$ in people with frequent healthcare utilization to $22.6 \%$ in people with negative self-rated health). All patterns of NCDs were significantly associated to all adverse health outcomes. Specifically, while the pattern "respiratory conditions" was more strongly associated with negative self-rated health and short-term disability, the pattern "RMDs and depression" was the most strongly associated with chronic pain (adjPR 2.04, 95\% CI 1.87-2.23). All NCDs patterns showed similar strength of association across the remaining outcomes. Figure 4 shows that patterns "cardiometabolic conditions" and "RMDs and depression" had similar PAF estimates across all adverse health outcomes. Those patterns had the highest PAF estimates for negative self-rated health $(16.0 \%, 95 \%$ CI 14.3-18.0, and 14.5\%, 95\% CI 13.0-16.0; respectively). Additionally, the "RMDs and depression" pattern showed significantly higher PAF estimates for chronic pain (PAF 8.9\%, 95\% CI 7.6-10.3) than the "cardiometabolic conditions" and "respiratory conditions" patterns. 


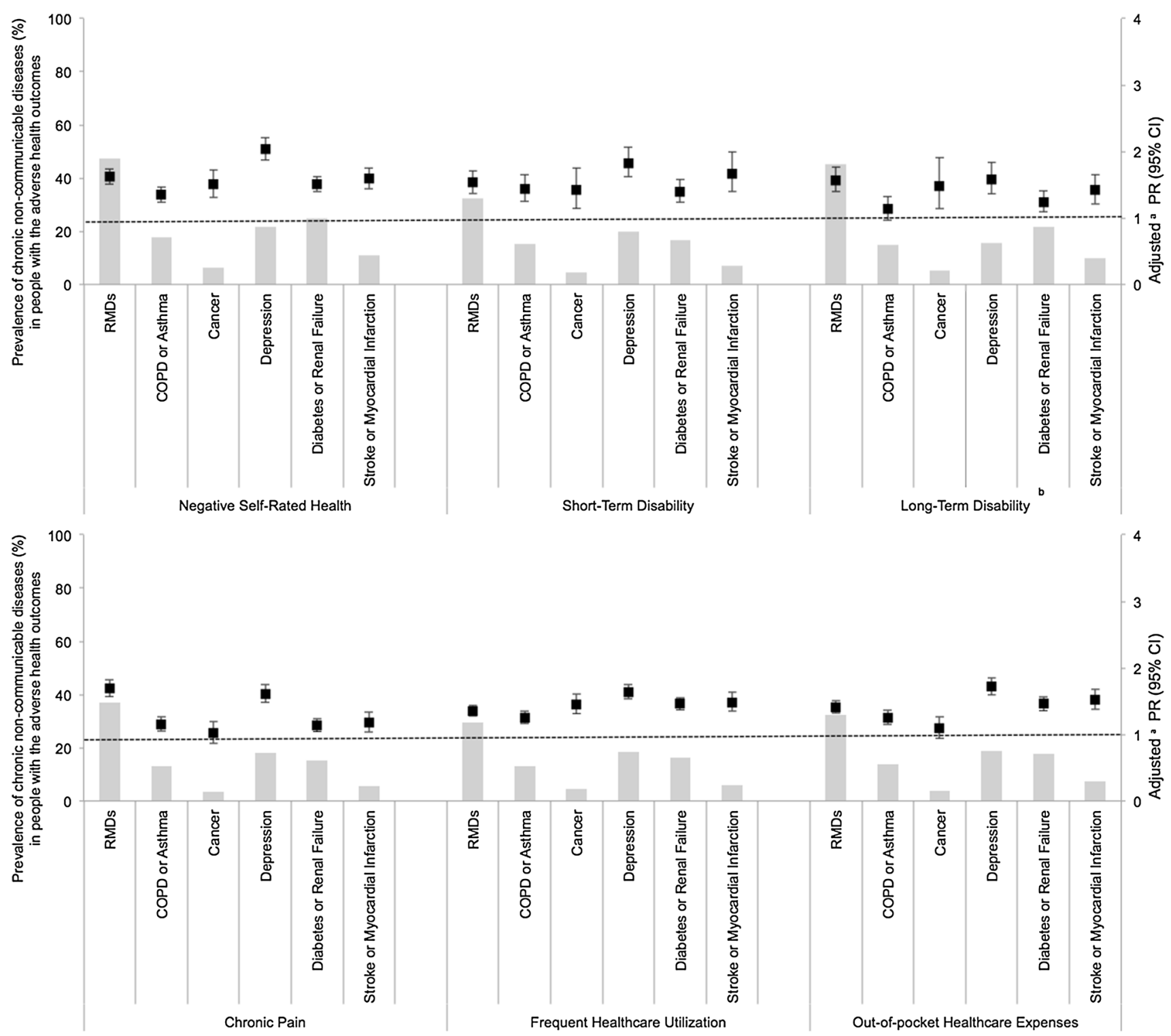

Fig. 1 Prevalence of GBD-defined groups of non-communicable diseases in people with the adverse health outcome (bars) and corresponding adjusted prevalence ratios (squares) with 95\% confidence intervals in the general population. Fourth National Health Survey (IV-INS), Portugal, 2005-2006. GDB global burden of disease, $R M D s$ rheumatic and musculoskeletal diseases, $C O P D$ chronic

\section{Discussion}

Our population-level results show that, using GBD-defined groups, RMDs had the highest impact among all diseases considered on all adverse health outcomes after adjustment to the remaining NCDs. When looking at empirical multimorbidity patterns, the "cardiometabolic conditions" (38.6\% probability of RMDs) and "RMDs and depression" (66.7\% RMDs) patterns had the highest and similar PAF obstructive pulmonary disease, $P R$ prevalence ratio, 95\% CI 95\% confidence interval. ${ }^{\text {a }} \mathrm{PR}$ adjusted for sex, age, education and presence of other GBD-defined groups of non-communicable diseases. ${ }^{\mathrm{b}} \mathrm{Col}-$ lected only in one trimester, due to logistic restrictions. All data are weighted

estimates, except for chronic pain, where the "RMDs and depression" pattern had the highest impact.

Our paper reveals important data on the population impact of RMDs in the context of realistic clusters of multimorbidity. For context, we compare the results with those obtained using a standard disease adjustment method, i.e., GBD-defined groups. In the GBD-defined groups approach we found that RMDs were the most prevalent conditions, while depression had the highest magnitude of association with all the adverse health outcomes. Consequently, these 
Fig. 2 Population attributable fractions (\%) and 95\% confidence intervals for each adverse health outcome by GBD-defined groups of non-communicable diseases in the general population. Fourth national health survey (IV-INS), Portugal, 2005-2006. GDB global burden of disease, $P A F$ population attributable fraction, $95 \% \mathrm{CI}$ 95\% confidence interval, $R M D s$ Rheumatic and musculoskeletal diseases, $C O P D$ chronic obstructive pulmonary disease. ${ }^{\mathrm{a} C}$ Collected only in one trimester, due to logistic restrictions

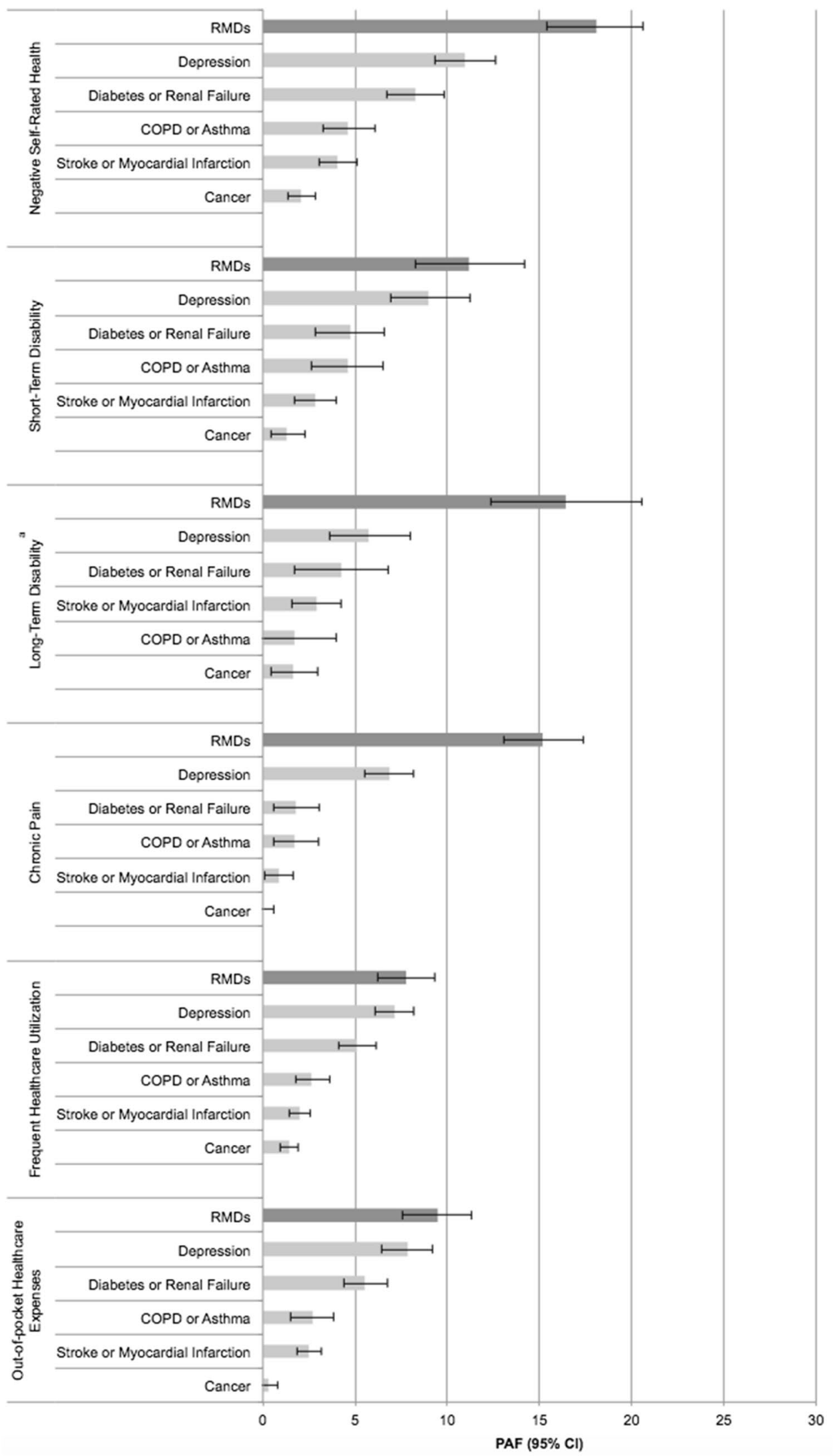



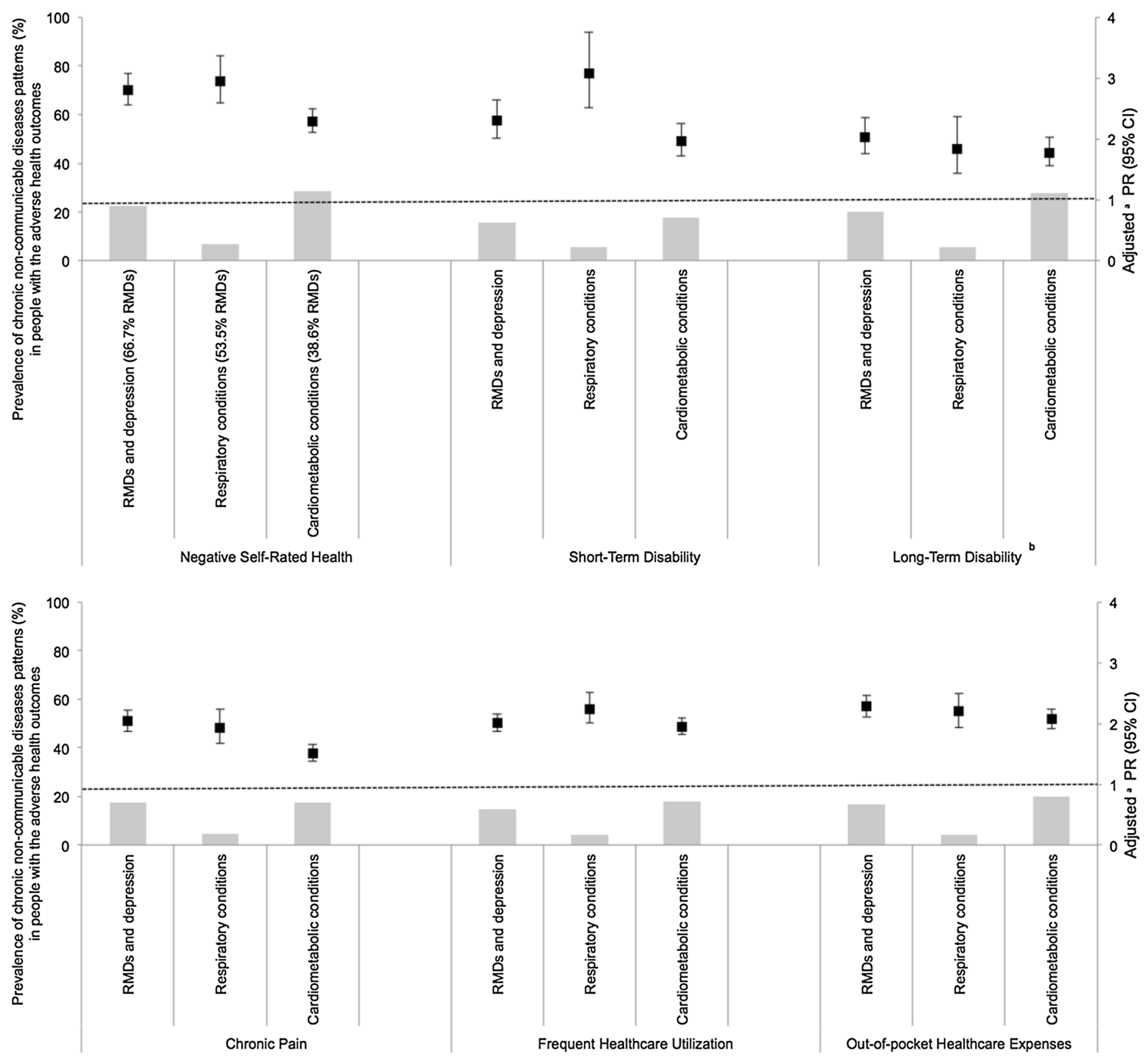

Fig. 3 Prevalence of non-communicable diseases patterns in people with the adverse health outcome (bars) and corresponding adjusted prevalence ratios (squares) with $95 \%$ confidence intervals in the general population. Fourth national health survey (IV-INS), Portugal, 2005-2006. RMDs rheumatic and musculoskeletal diseases, COPD

two conditions, and especially RMDs, show the highest PAF for adverse outcomes. Our second approach, using empirical multimorbidity patterns, shows that the "cardiometabolic conditions" pattern was the most prevalent, followed by "RMDs and depression". PAF estimates for adverse health outcomes were generally similar between those patterns.

The differences found using different approaches may be explained by conceptual issues. Our approach to deal with the classic GBD-defined groups accounts for the coexistence of multiple conditions in a multivariate model. This type chronic obstructive pulmonary disease, $P R$ prevalence ratio, $95 \% C I$ $95 \%$ confidence interval. ${ }^{\text {a }} \mathrm{PR}$ adjusted for sex, age and education. ${ }^{\mathrm{b}}$ Collected only in one trimester, due to logistic restrictions. Reference: "low disease probability" pattern (7.8\% probability of RMDs). All data are weighted

of essentially frequentist approach is very commonly used to solve confounding issues and probably easier to implement in most statistical packages. However, this approach is unrealistic since not all of the possible disease combinations occur in the population. For this reason, the interpretation of PAF obtained from adjusted models becomes challenging and may lose meaning at the individual level or produce bias in effect estimates for each disease. In contrast, empirical mutually exclusive patterns of NCDs are specifically designed to capture disease co-occurrence rather than 
Fig. 4 Population attributable fractions (\%) and $95 \%$ confidence intervals for each adverse health outcome by non-communicable diseases patterns in the general population. Fourth National Health Survey (IV-INS), Portugal, 2005-2006. PAF population attributable fraction, $95 \% \mathrm{CI}$ 95\% confidence interval, $R M D$ s rheumatic and musculoskeletal diseases. ${ }^{\mathrm{a}}$ Collected only in one trimester, due to logistic restrictions. Reference: "Low disease probability" pattern $(7.8 \%$ probability of RMDs)

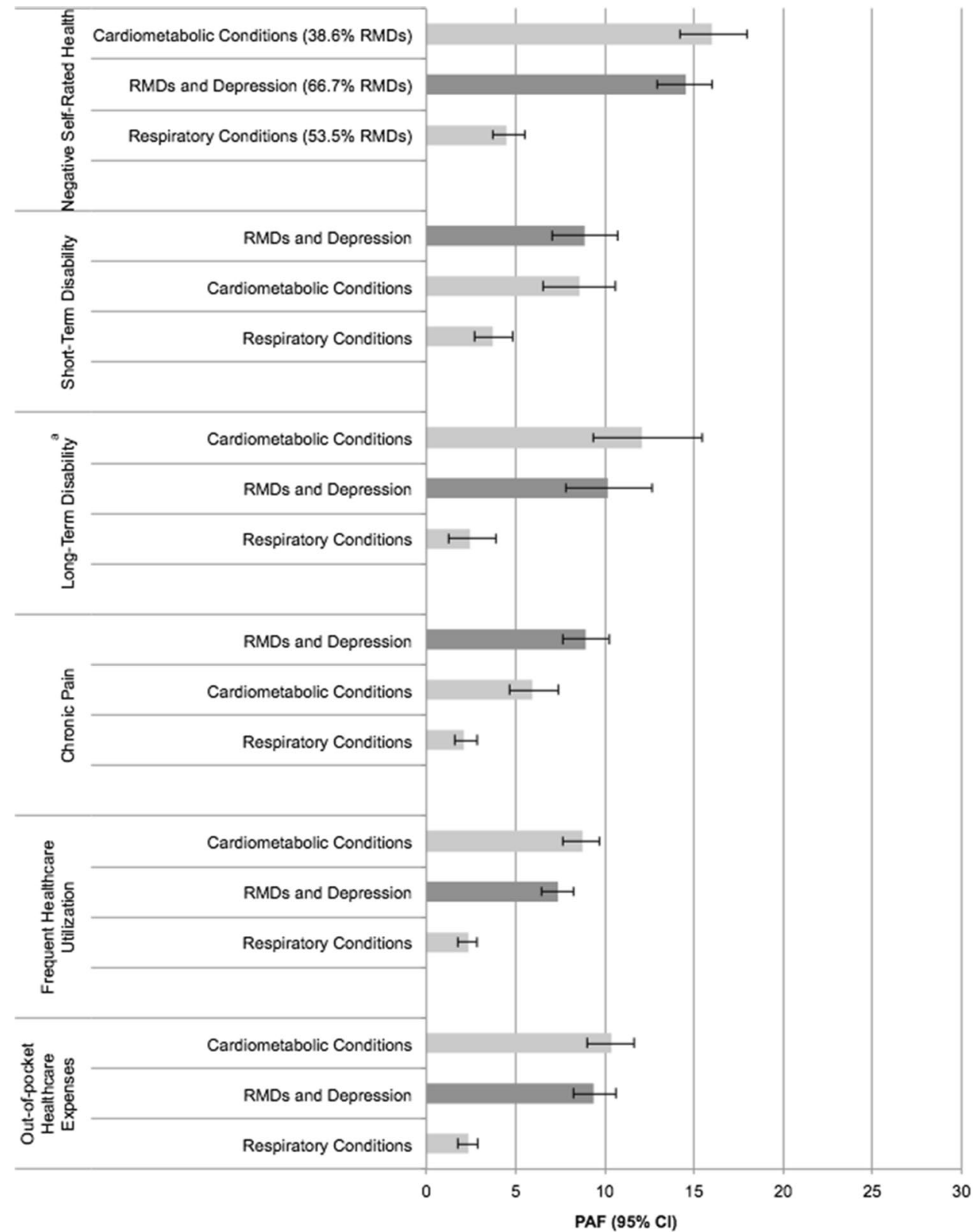

dealing with it as a problem of confounding. This conveys a more realistic picture of multimorbidity beyond estimating "independent effects" but requires more complex statistical analysis as well as a priori knowledge and assumptions regarding the number and meaning of disease patterns. Due to their empirical nature, patterns emerge only when they occur in the population, regardless of all theoretically possible combinations of diseases. The classification of individuals is then based on the most likely class assignment, meaning that each individual is assigned to a single pattern with a specific set of probability distributions for different diseases. In this context patterns are interpreted as mutually exclusive with regard to the assignment of individuals, but the same disease may (and does) occur in more than one pattern, e.g., even though RMDs were more likely in subjects assigned to the pattern "RMDs and depression" (66.7\%), an RMD probability of $38.6 \%$ still exists in the "cardiometabolic conditions" pattern. Overall, the population impact of NCDs seems to be comparatively overestimated when considering the traditional GBD-defined groups, since in the latter approach PAF interpretation assumes the complete and irreversible elimination of an NCD or group, while in the empirical approach PAF interpretation considers the change of class membership into the "low disease probability" pattern, which still has some disease probability (including a $7.8 \%$ prevalence of RMDs) [15]. Whereas, in our first approach exposure was defined deterministically, since all individuals reported who RMDs were classified as exposed, in our 
second approach exposure was defined as the most likely pattern, meaning that individuals exposed to the "RMDs and depression" pattern had a $66.7 \%$ probability of reporting RMDs. This may result in a relative underestimation of PAF in the second approach.

We found strong associations between negative self-rated health and several GBD-based diseases (depression, RMDs, and stroke or myocardial infarction), as well as our empirical multimorbidity patterns. These results are congruent with previous inverse associations between self-rated health and depression, osteoarthritis, and heart failure [17], and the general group of RMDs [18], or even with the multimorbidity patterns [19]. When looking at impact measures, the largest PAF was found for RMDs (18.1\%), and this finding is similar to those previously described, namely a PAF of $15.5 \%$ for arthritis [2] and a population attributable risk of $13.0 \%$ for isolated rheumatoid arthritis [4].

Our findings regarding disability are also in accordance with previous studies, both at the individual and population levels [2,3], reporting that participants with RMDs have a higher risk of long-term disability, as well as an increased risk of activity limitations [20]. Moreover, having a RMD in co-occurrence with other conditions seems to worsen daily functioning [21], which is sustained by our empirical approach.

Despite the fact that chronic pain is frequently addressed as a condition in itself our data support that it is indeed a bigger concern in RMDs in comparison to other conditions [22], both in terms of the magnitude of association (adjPR 1.69) and with regard to the fraction of chronic pain in the population that might be attributed to RMDs (PAF 15.2\%).

Regarding resource use several studies have reported RMDs as one of the main causes of frequent healthcare utilization [23, 24]. Although our GBD-based approach suggested a weak association between RMDs and healthcare utilization in comparison with other chronic conditions, a high population impact was found. This suggests that subjects with severe clinical conditions, like stroke and myocardial infarction, seem to be more likely to report resource use outcomes but, due to the high prevalence of RMDs, the latter overcome those conditions in terms of population impact. A similar result was found for out-of-pocket healthcare expenses. Likewise, our empirical approach supports that RMDs are a major driver of resource use and this is concordant with previous evidence [25].

Some methodological issues should be addressed. The self-reported nature of NCDs classification, as well as the specific validation question used in the survey ("Was it a doctor or a nurse who told you that you had that disease?"), could under- or over-estimate disease prevalence and respective population impact. Despite that, health surveys seem to be more accurate to detect RMDs and other conditions that might be undervalued in clinical practice and thus underestimated in studies based on clinical records [26]. The institutionalized population was excluded from the sampling frame, which might have caused an underestimation of the frequency of the most severe cases of disease, although it is not clear whether associations or impact on adverse health outcomes would be affected. Our outcomes were selected fulfilling the OMERACT consensus group recommendation, which was specifically designed to address clinical characteristics of RMDs, and this may have over-valued their impact when compared to other NCDs. Additionally, in accordance with the GBD list of conditions, hypertension and osteoporosis are not considered as diseases per se, but instead classified as risk factors for cardiovascular conditions and RMDs [27, 28], respectively. This may have decreased the true population impact of cardiovascular conditions and RMDs. The inclusion of hypertension and osteoporosis in our second empirical approach may therefore comparatively overestimate the population impact of multimorbidity patterns. Finally, the population burden could be further explored with a breakdown of diseases or disease groups by etiology, disease duration or severity, but the IV-INS has limited data in that respect. Nevertheless, we expect that most RMDs in our population-based adult sample will have a degenerative etiology, even though we have no definitive validation of this assumption. This may be helpful for the interpretation of our results, but requires prudence regarding inference about the impact of conditions with an inflammatory etiology.

Despite these methodological issues, some strengths are evident. This study included data from a national representative health survey with a large sample size and a wide range of ages. Additionally, in an effort to enhance international comparability, we adopted acknowledged frameworks for selecting NCDs [13], and specific adverse health outcomes $[8,9]$. We also computed PAF applying the recommended adjusted formula [29-31] and using prevalence ratios instead of odds ratios as appropriated when the outcome of interest is frequent in the population [2]. We also present confidence intervals for impact measures, which improve comparisons between estimates and are frequently unreported [31]. Additionally, our empirical model-based patterns of NCDs provide unique and valuable information for public health planning, namely for the prioritization of population-level interventions for the prevention and management of NCDs, and specifically to address the high burden of RMDs. The patterns approach is expectedly more meaningful since it better represents a patient-oriented model, by means of estimating a realistic impact of RMDs in the context of their high co-occurrence with other NCDs.

In conclusion, regardless of using a standard adjustment or an empirical pattern approach, RMDs revealed a great population impact across all the adverse health outcomes, especially chronic pain. This reinforces that integrated 
public health programs targeting common risk factors for RMDs and other NCDs may be synergistic in improving population health.

Acknowledgements The authors thank the National Health Institute Doutor Ricardo Jorge (INSA), the Ministry of Health, and Statistics Portugal (INE, IP) for providing the data. They also kindly acknowledge the contribution of Ana Isabel Ribeiro in the revision of the manuscript.

Author contributions All authors were involved in drafting the article or revising it critically for important intellectual content, and all authors approved the final version to be submitted for publication. DS, TM and RL designed the study. DS performed the statistical analyses and drafted the first manuscript with input from all the other authors.

Funding This study received no specific funding. The funding for EPI Unit is obtained from the National Foundation for Science and Technology (FCT UID/DTP/04750/2013/002). FAA is supported by Grant FCT SFRH/BD/85398/2012, TM by Grant FCT SFRH/BD/92370/2013 and RL by Grant FCT SFRH/BPD/88729/2012.

\section{Compliance with ethical standards}

Conflict of interest All authors have declared that no conflict of interest exists.

Ethical approval The Portuguese National Health Survey is currently conducted in accordance with the European and national legal framework that stems from the Regulation (EC) No 1338/2008 of the European Parliament and of the Council of 16 December 2008 on Community statistics on public health and health and safety at work, through the national "Lei 22/2008 de 13 de maio". Ethical standards in data collection and individual data protection are safeguarded by national and international legislation and are the responsibility of the entities that conduct the survey. The authors of the present paper were granted access to the anonymized data and take responsibility for the present study.

\section{References}

1. Woolf AD, Vos T, March L (2010) How to measure the impact of musculoskeletal conditions. Best Pract Res Clin Rheumatol 24(6):723-732. https://doi.org/10.1016/j.berh.2010.11.002

2. Perruccio AV, Power JD, Badley EM (2007) The relative impact of 13 chronic conditions across three different outcomes. J Epidemiol Community Health 61(12):1056-1061. https://doi.org/10.1136/ jech.2006.047308

3. Palazzo C, Ravaud JF, Trinquart L, Dalichampt M, Ravaud P, Poiraudeau S (2012) Respective contribution of chronic conditions to disability in France: results from the national disabilityhealth survey. PLoS One 7(9):e44994. https://doi.org/10.1371/ journal.pone.0044994

4. Molarius A, Janson S (2002) Self-rated health, chronic diseases, and symptoms among middle-aged and elderly men and women. J Clin Epidemiol 55(4):364-370

5. Griffith L, Raina P, Wu H, Zhu B, Stathokostas L (2010) Population attributable risk for functional disability associated with chronic conditions in Canadian older adults. Age Ageing 39(6):738-745. https://doi.org/10.1093/ageing/afq105
6. Davis CH, MacKinnon DP, Schultz A, Sandler I (2003) Cumulative risk and population attributable fraction in prevention. J Clin Child Adolesc Psychol 32(2):228-235. https://doi. org/10.1207/s15374424jccp3202_7

7. Hoy DG, Smith E, Cross M, Sanchez-Riera L, Blyth FM, Buchbinder R, Woolf AD, Driscoll T, Brooks P, March LM (2015) Reflecting on the global burden of musculoskeletal conditions: lessons learnt from the global burden of disease 2010 study and the next steps forward. Ann Rheum Dis 74(1):4-7. https://doi. org/10.1136/annrheumdis-2014-205393

8. Boers M, Kirwan JR, Wells G, Beaton D, Gossec L, d'Agostino MA, Conaghan PG, Bingham CO 3rd, Brooks P, Landewe R, March L, Simon LS, Singh JA, Strand V, Tugwell P (2014) Developing core outcome measurement sets for clinical trials: OMERACT filter 2.0. J Clin Epidemiol 67 (7):745-753. https ://doi.org/10.1016/j.jclinepi.2013.11.013

9. Kirwan JR, Boers M, Hewlett S, Beaton D, Bingham CO III, Choy E, Conaghan PG, D'Agostino MA, Dougados M, Furst DE, Guillemin F, Gossec L, van der Heijde DM, Kloppenburg M, Kvien TK, Landewe RB, Mackie SL, Matteson EL, Mease PJ, Merkel PA, Ostergaard M, Saketkoo LA, Simon L, Singh JA, Strand V, Tugwell P (2014) Updating the OMERACT filter: core areas as a basis for defining core outcome sets. J Rheumatol 41(5):994-999. https://doi.org/10.3899/jrheum.131309

10. Radner H (2016) Multimorbidity in rheumatic conditions. Wien Klin Wochenschr 128(21-22):786-790. https://doi.org/10.1007/ s00508-016-1090-x

11. Hilderink HB, Plasmans MH, Snijders BE, Boshuizen HC, Poos MJ, van Gool CH (2016) Accounting for multimorbidity can affect the estimation of the Burden of Disease: a comparison of approaches. Arch Public Health 74:37. https://doi.org/10.1186/ s13690-016-0147-7

12. INE, INSA (2009) Inquérito Nacional de Saúde 2005/2006. Instituto Nacional de Estatística, Instituto Nacional Ricardo Jorge

13. Goodman RA, Posner SF, Huang ES, Parekh AK, Koh HK (2013) Defining and measuring chronic conditions: imperatives for research, policy, program, and practice. Prev Chronic Dis 10:E66. https://doi.org/10.5888/pcd10.120239

14. Vos T et al (2012) Years lived with disability (YLDs) for 1160 sequelae of 289 diseases and injuries 1990-2010: a systematic analysis for the Global Burden of Disease Study 2010. Lancet 380(9859):2163-2196. https://doi.org/10.1016/s0140 $-6736(12) 61729-2$

15. Simões D, Araújo FA, Severo M, Monjardino T, Cruz I, Carmona L, Lucas R (2017) Patterns and consequences of multimorbidity in the general population: there is no chronic disease management without rheumatic disease management. Arthritis Care Res 69(1):12-20. https://doi.org/10.1002/acr.22996

16. Rust KF, Rao JN (1996) Variance estimation for complex surveys using replication techniques. Stat Methods Med Res 5(3):283-310

17. Tinetti ME, McAvay GJ, Chang SS, Newman AB, Fitzpatrick AL, Fried TR, Peduzzi PN (2011) Contribution of multiple chronic conditions to universal health outcomes. J Am Geriatr Soc 59(9):1686-1691

18. Badley EM, Kasman NM (2004) The impact of arthritis on Canadian women. BMC Womens Health 4(Suppl 1):S18. https://doi. org/10.1186/1472-6874-4-s1-s18

19. Gunn JM, Ayton DR, Densley K, Pallant JF, Chondros P, Herrman HE, Dowrick CF (2012) The association between chronic illness, multimorbidity and depressive symptoms in an Australian primary care cohort. Soc Psychiatry Psychiatr Epidemiol 47(2):175-184. https://doi.org/10.1007/s00127-010-0330-z

20. Slater M, Perruccio AV, Badley EM (2011) Musculoskeletal comorbidities in cardiovascular disease, diabetes and respiratory disease: the impact on activity limitations; a representative 
population-based study. BMC Public Health 11:77. https://doi. org/10.1186/1471-2458-11-77

21. Loza E, Jover JA, Rodriguez L, Carmona L (2009) Multimorbidity: prevalence, effect on quality of life and daily functioning, and variation of this effect when one condition is a rheumatic disease. Semin Arthritis Rheum 38(4):312-319. https://doi.org/10.1016/j. semarthrit.2008.01.004

22. Scherer M, Hansen H, Gensichen J, Mergenthal K, Riedel-Heller S, Weyerer S, Maier W, Fuchs A, Bickel H, Schon G, Wiese B, Konig HH, van den Bussche H, Schafer I (2016) Association between multimorbidity patterns and chronic pain in elderly primary care patients: a cross-sectional observational study. BMC Fam Pract 17:68. https://doi.org/10.1186/s12875-016-0468-1

23. Badley EM, Rasooly I, Webster GK (1994) Relative importance of musculoskeletal disorders as a cause of chronic health problems, disability, and health care utilization: findings from the 1990 Ontario Health Survey. J Rheumatol 21(3):505-514

24. Andrianakos AA, Miyakis S, Trontzas P, Kaziolas G, Christoyannis F, Karamitsos D, Karanikolas G, Dantis P (2005) The burden of the rheumatic diseases in the general adult population of Greece: the ESORDIG study. Rheumatology 44(7):932-938. https ://doi.org/10.1093/rheumatology/keh650

25. Glynn LG, Valderas JM, Healy P, Burke E, Newell J, Gillespie P, Murphy AW (2011) The prevalence of multimorbidity in primary care and its effect on health care utilization and cost. Fam Pract 28(5):516-523. https://doi.org/10.1093/fampra/cmr013

26. Violan C, Foguet-Boreu Q, Hermosilla-Perez E, Valderas JM, Bolibar B, Fabregas-Escurriola M, Brugulat-Guiteras P, Munoz-Perez MA (2013) Comparison of the information provided by electronic health records data and a population health survey to estimate prevalence of selected health conditions and multimorbidity. BMC Public Health 13:251. https://doi. org/10.1186/1471-2458-13-251
27. Forouzanfar MH, Liu P, Roth GA, Ng M, Biryukov S, Marczak L, Alexander L, Estep K, Hassen Abate K, Akinyemiju TF, Ali R, Alvis-Guzman N, Azzopardi P, Banerjee A, Barnighausen T, Basu A, Bekele T, Bennett DA, Biadgilign S, Catala-Lopez F, Feigin VL, Fernandes JC, Fischer F, Gebru AA, Gona P, Gupta R, Hankey GJ, Jonas JB, Judd SE, Khang YH, Khosravi A, Kim YJ, Kimokoti RW, Kokubo Y, Kolte D, Lopez A, Lotufo PA, Malekzadeh R, Melaku YA, Mensah GA, Misganaw A, Mokdad AH, Moran AE, Nawaz H, Neal B, Ngalesoni FN, Ohkubo T, Pourmalek F, Rafay A, Rai RK, Rojas-Rueda D, Sampson UK, Santos IS, Sawhney M, Schutte AE, Sepanlou SG, Shifa GT, Shiue I, Tedla BA, Thrift AG, Tonelli M, Truelsen T, Tsilimparis N, Ukwaja KN, Uthman OA, Vasankari T, Venketasubramanian N, Vlassov VV, Vos T, Westerman R, Yan LL, Yano Y, Yonemoto N, Zaki ME, Murray CJ (2017) Global burden of hypertension and systolic blood pressure of at least 110 to $115 \mathrm{~mm} \mathrm{Hg}, 1990-2015$. JAMA 317 (2):165-182. https://doi.org/10.1001/jama.2016.19043

28. Sanchez-Riera L, Carnahan E, Vos T, Veerman L, Norman R, Lim SS, Hoy D, Smith E, Wilson N, Nolla JM, Chen JS, Macara M, Kamalaraj N, Li Y, Kok C, Santos-Hernandez C, March L (2014) The global burden attributable to low bone mineral density. Ann Rheum Dis 73(9):1635-1645. https://doi.org/10.1136/annrheumdi s-2013-204320

29. Darrow LA, Steenland NK (2011) Confounding and bias in the attributable fraction. Epidemiology 22(1):53-58. https://doi. org/10.1097/EDE.0b013e3181fce49b

30. Rockhill B, Newman B, Weinberg C (1998) Use and misuse of population attributable fractions. Am J Public Health 88(1):15-19

31. Williamson DF (2010) The population attributable fraction and confounding: buyer beware. Int J Clin Pract 64(8):1019-1023. https://doi.org/10.1111/j.1742-1241.2010.02443.x 